La Revue

des Droits

de l'Homme

\section{La Revue des droits de l'homme}

Revue du Centre de recherches et d'études sur les droits fondamentaux

$1 \mid 2012$

Revue des droits de l'homme $-\mathrm{N}^{\circ} 1$

\title{
Chapitre 2. La responsabilité sociale des entreprises
}

À mi-chemin entre la soft law et le jus cogens : la question de l'effectivité de la protection des droits sociaux par les entreprises multinationales

\section{Claire Marzo}

\section{OpenEdition}

\section{Journals}

Édition électronique

URL : http://journals.openedition.org/revdh/153

DOI : $10.4000 /$ revdh. 153

ISSN : 2264-119X

\section{Éditeur}

Centre de recherches et d'études sur les droits fondamentaux

\section{Édition imprimée}

Date de publication : 1 juin 2012

Pagination : 409-425

Référence électronique

Claire Marzo, "Chapitre 2. La responsabilité sociale des entreprises ", La Revue des droits de l'homme [En ligne], 1 | 2012, mis en ligne le 30 juin 2012, consulté le 08 juillet 2020. URL : http:// journals.openedition.org/revdh/153; DOI : https://doi.org/10.4000/revdh.153 


\section{CHAPITRE 2 -}

\section{LA RESPONSABILITE SOCIALE DES ENTREPRISES.}

\section{A MI-CHEMIN ENTRE LA SOFT LAW ET LE JUS COGENS : LA QUESTION DE L'EFFECTIVITE DE LA PROTECTION DES DROITS SOCIAUX PAR LES ENTREPRISES MULTINATIONALES}

\section{Claire MARZO}

C'est un lieu commun que de dire que les droits fondamentaux et en particulier les droits sociaux sont des droits opposables aux personnes publiques ${ }^{1139}$. Il est, par contre, beaucoup moins admis que des acteurs non étatiques puissent se voir chargés de la mise en œuvre de droits sociaux. Les débiteurs des droits sociaux sont le plus souvent des États mais on trouve encore des personnes privées: des personnes physiques, par exemple dans le cadre du droit de la famille français ${ }^{1140}$, ou des personnes morales comme les entreprises par le biais de la responsabilité sociale des entreprises (RSE).

Les prémices de cette affirmation reposent sur la théorie de la citoyenneté sociale au sens des lois Auroux sur la démocratie industrielle ${ }^{1141}$. L'idée était que les droits sociaux devaient transpercer l'unité juridique de l'entreprise pour s'appliquer aussi aux travailleurs. Alors qu'auparavant seule l'entreprise se voyait accorder des droits et devoirs, il fallait décomposer plus avant cette entité afin de protéger les salariés face aux employeurs. Cela s'est traduit par la reconnaissance de droits procéduraux et matériels aux salariés. Lors d'un passage au niveau international, principalement lié à la mondialisation ${ }^{1142}$, l'idée d'une protection des travailleurs n'a pas décru ${ }^{1143}$. Mais elle s'est trouvée lésée face à une augmentation des pouvoirs de déplacement

Une version plus complète de ce rapport est disponible en ligne: http://droits-sociaux.uparis10.fr/assets/files/rapport final/RSE claire marzo.pdf.

1139 Avec les controverses qu’on lui connaît... V. Joël ANDRIANTSIMBAZOVINA, Hélène GAUDIN, Jean-Pierre MARGUENAUD, Stéphane RIALS, François SUDRE, Dictionnaire des droits de l'Homme, Paris, Dunod / PUF, 2008.

1140 V. Marc PICHARD, infra.

1141 Martine LE FRIANT, " La démocratie sociale, entre formule et concept ", Regards, 2001, vol. 19, pp. 47-53, p. 6.

1142 Définie comme l'« intensification des relations à travers le monde qui relient des localités distantes d'une telle manière que des évènements locaux sont engendrés par des causes géographiquement lointaines et vice versa ", V. Jürgen HABERMAS, " The European nation state: Its achievements and its limitations, on the past and future of sovereignty and citizenship ", Ratio Juris, 1996, 9(2), pp. 125-137.

1143 Franck COCHOY, "La responsabilité sociale de l'entreprise comme "représentation" de l'économie et du droit ", Droit et Société, 01/01/2007, n 65, pp. 91 -101. 
géographique de l'entreprise et à l'affaiblissement controversé de l'État face à ces mouvements.

Deux phénomènes ont donc conduit à la RSE : l'intégration des droits sociaux au sein de l'entreprise et le retrait progressif de la mainmise de l'État sur la question de la protection des droits sociaux. Ces deux phénomènes se sont croisés pour conduire à une protection bigarrée et parfois limitée des droits sociaux.

Pourtant, la principale spécificité de la RSE est son caractère volontaire ou non obligatoire, son habit de droit mou (soft law). L'entreprise s'engage seule et sans contrepartie envers ses travailleurs, mais ne se contraint pas. Définie par la Commission européenne comme "l'intégration volontaire par les entreprises de préoccupations sociales et environnementales à leurs activités commerciales et leurs relations avec leurs parties prenantes »1144, elle trouve parfois des définitions plus étroites ou plus larges. Par exemple, selon Milton Friedman, elle «se limite à la création de profits et d'emplois, au paiement des impôts et au respect des règles du jeu définies par les pouvoirs publics » ${ }^{1145}$. D'autres proposent encore une définition hiérarchisée insinuant que "les pratiques de RSE s'ajoutent à celles qui visent à créer des profits, ce qui conduit à une hiérarchisation entre la responsabilité économique et sociale des entreprises, cette dernière constituant un luxe que l'on pourrait s'offrir dans les périodes de croissance économique ${ }^{1146}$. Quelle que soit la définition choisie, le degré important de choix de l'entreprise de s'engager volontairement pour la protection des droits sociaux de leurs travailleurs et d'autres parties prenantes apparaît clairement. "Cette approche ouvre les perspectives les plus intéressantes pour l'entreprise et la société et semble pouvoir convaincre les dirigeants de s'engager durablement dans des démarches de RSE ambitieuses $» 1147$.

Cette notion, très décriée à ses débuts, a connu depuis un certain essor $^{1148}$. Elle se traduit concrètement par de nombreux types de textes, les plus renommés étant les codes de conduite ${ }^{1149}$. Elle conduit à la protection de droits sociaux variés ${ }^{1150}$. Elle soulève un grand nombre de questions : quelle mise en œuvre des droits sociaux? par qui ? quels contrôles? par quels mécanismes ${ }^{1151}$ ?

\footnotetext{
${ }^{1144} \operatorname{COM}(2002) 347$ final et $\operatorname{COM}(2006) 136$ final.

1145 André SOBCZAK, "Pour une définition de la RSE à la hauteur des enjeux !", Metis, 16 Avril 2010, http://www.metiseurope.eu/pour-une-definition-de-la-rse-a-la-hauteur-desenjeux_fr_70_art_28765.html.

1146 André SOBCZAK propose d’intégrer les comportements obligatoires, ibid.

1147 Ibid.

1148 Par exemple, http://www.fidh.org/Entreprises-et-Droits-de-l-Homme-Un-guide-sur-les.

1149 V. Fiona McLEAY, "Corporate Codes of Conduct and the Human Rights Accountability of Transnational Corporations : A Small Piece of a Larger Puzzle ", in Olivier DE SCHUTTER, Transnational corporations and human rights, Oxford / Portland, Oregon, Hart Pub., 2006, pp. 219-240. 1150 V. infra, partie I.

1151 V. Claire MARZO, "Les risques juridiques créés par les accords-cadres internationaux (opportunités / dangers / stratégies ", in Marie-Ange MOREAU Justice et mondialisation du droit
} 
De façon plus normative, quelles évolutions peut-on en attendre? Faut-il contraindre les entreprises ? Et le peut-on ? Quel est le rôle des organisations internationales ? L'Europe connaît-elle des particularités ? La décentralisation de la protection des droits est-elle une bonne chose ${ }^{1152}$ ? De nombreux auteurs ont tenté de répondre à certaines de ces questions ${ }^{1153}$. L'objectif de cette étude se limite à l'une d'entre elles: celle de l'effectivité de la protection des droits sociaux par les entreprises multinationales.

L'intérêt de cette question réside dans un paradoxe qui réside dans la définition même de la RSE. Elle a pour objectif la contrainte de l'entreprise au respect de règles de droit social. Pourtant, son caractère volontaire rend l'idée d'une contrainte (étatique ou non) impossible. En dehors de l'État, le socle étatique ou international de valeurs communes risque de ne plus être respecté et le travailleur peut se trouver dans un état de quasi-incapacité à se défendre. L'entreprise se retrouve toute puissante. Et l'État inutile. La question de la contrainte de l'entreprise toute puissante est posée. Ce paradoxe empêche d'identifier une solution claire. On se tourne plutôt vers plusieurs stratégies de détournement des normes existantes. L'objectif est de contraindre une entreprise à respecter les engagements qu'elle a seule décidé de prendre.

L'approche choisie ici propose non plus de distinguer entre droit mou et droit dur, mais d'identifier une échelle des pratiques allant de la contrainte légale à l'invitation. Certains ont pu parler de droit matriciel ${ }^{1154}$. Ainsi, à la question de l'effectivité de la protection des droits sociaux par les entreprises multinationales, la réponse doit être double en ce qu'on trouve une protection directe et une protection indirecte.

Plus précisément, les droits sociaux peuvent être protégés par des organisations internationales, des États, et maintenant des entreprises - on parle d'opposabilité directe. Les organisations internationales et les États peuvent à leur tour chercher à s'assurer de la protection des droits sociaux au sein des entreprises: on trouve alors une protection et une opposabilité indirecte. Parce que la RSE est à mi-chemin entre la soft law et le jus cogens, nous traiterons de son opposabilité directe de droit mou (I), puis de son opposabilité indirecte de droit plus ou moins dur (II).

\section{Une opposabilite directe de droit mou}

La RSE consiste précisément en la possibilité pour les entreprises de prendre des engagements volontaires (A) et de les mettre en œuvre (B).

du travail, Conférence organisée en collaboration par l'université Paris I Panthéon-Sorbonne et l'IUE, à paraître 2010.

1152 On pensera à la théorie de la relativité des droits de l'Homme proposée par Alain SUPIOT, Homo juridicus : essai sur la fonction anthropologique du droit, Le Seuil, 2007.

${ }^{1153}$ Pour une bibliographie, v. la version longue de cet article, note 1.

${ }^{1154}$ Le droit à un travail décent permet de donner de la force à des plus petits droits et en tout cas à des programmes. V. infra. 


\section{A. Des engagements volontaires}

Les engagements volontaires des entreprises en matière de RSE sont de natures variées et comprennent une large gamme de droits sociaux. Il faut distinguer entre deux comportements distincts: le modèle anglo-saxon de responsabilité sociétale et le modèle continental. Ces conceptions diffèrent quant à la nature et au contenu des droits protégés ${ }^{1155}$.

1) Des instruments variés: Des codes de conduite aux accords cadres internationaux

L'intérêt de la RSE pour les entreprises tient principalement à son caractère volontaire. Elles ont la possibilité, si elles le souhaitent, de s'engager à respecter des droits sociaux et environnementaux. On comprendrait tout à fait que cette tendance ne trouve pas d'écho et soit peu développée puisqu'elle ne repose sur aucune obligation et qu'elle pourrait conduire à des frais rendant l'objectif de performance de l'entreprise difficile à atteindre.

Pourtant, ce phénomène auparavant ponctuel et limité à certaines grandes entreprises a trouvé à se répandre conduisant à une extension de son champ d'application. Il trouve aujourd'hui à se développer par trois biais : Le premier consiste en la reproduction volontaire par de plus petites entreprises, parfois nationales : on assiste à la reproduction de modèles donnés par les plus grandes entreprises par de plus petites ${ }^{1156}$.

Le second passe par la standardisation ${ }^{1157}$ dont les différentes techniques conduisent les acteurs d'un secteur à se plier aux normes en usage afin de faciliter les échanges. C'est un objectif pragmatique de simplification qui conduit à l'extension des pratiques.

Enfin, le troisième mouvement tient à une ouverture de ces pratiques à des acteurs plus diversifiés. Au départ limitée aux entreprises, la RSE s'adresse maintenant aussi aux organisations non gouvernementales qui semblent prêtes à s'impliquer davantage dans la coopération avec les États et les entreprises.

Le nombre et la diversité des textes augmentent en conséquence. Il y a quelques années, les codes de conduite constituaient la majeure partie des textes de RSE. Aujourd'hui, on trouve non seulement des simples déclarations d'intentions, mais aussi des instruments de standardisation ou même des accords négociés. Par exemple, les accords cadres internationaux qui constituent

1155 André HABISCH, Jan JONKER, Martina WEGNER, Corporate social responsibility across Europe, Berlin, Springer, 2005, p. 10.

1156 Aurora VOICULESCU, «Human Rights under the Corporate Sphere of Influence : Socialising Economic Relationships through Corporate Social Responsibility Processes ", in Bettina LANGE, Socializing Economic Relationships: New Perspectives and Methods for Analysing Transnational Risk Regulation, workshop, Oxford may 2010.

1157 Ce phénomène est encore analysé par Göran AHRNE, Nils BRUNSSON, "L’organisation en dehors des organisations, ou l'organisation incomplète », AEGIS le Libellio, pp. 1-20, p. 4-5. 
une nouvelle catégorie sont définis comme un accord entre une entreprise multinationale et une fédération syndicale internationale ayant pour objet les activités internationales de cette entreprise ${ }^{1158}$. Plusieurs classifications ont été proposées. On peut, par exemple, distinguer entre des codes internes ${ }^{1159}$ et des codes externes créés soit dans des instances multinationales (Nations Unies, Organisation internationale du travail), soit par un gouvernement ou une organisation non gouvernementale ${ }^{1160}$. Le niveau de complexité augmente, mais les objectifs de ces codes sont souvent les mêmes : ils cherchent à assurer une certaine protection des droits sociaux.

\section{2) La protection des droits sociaux}

Les droits protégés sont généralement des droits sociaux. Ils sont pourtant de différents ordres. On trouve aussi bien des droits procéduraux de citoyenneté industrielle au sens de Marshall - tels que le droit de s'organiser au sein de l'entreprise pour protéger ses droits sociaux, le dialogue social en Europe, la négociation collective dans chaque État, le dialogue avec les fédérations internationales de représentation des travailleurs dans quelques cas de droit international - que des droits matériels comme la reconnaissance des droits sociaux, le droit à la sécurité sociale, le droit au welfare, le droit au logement, à la santé ou à la sécurité ${ }^{1161}$.

De nombreux codes de conduites et accords font référence aux normes de l'Organisation internationale du travail (OIT) ${ }^{1162}$, parfois à sa déclaration de 1998 et à ses quatre droits fondamentaux - la prohibition du travail forcé, du travail des enfants, de la discrimination et la reconnaissance de la liberté d'association ${ }^{1163}$. D'autres droits se retrouvent aussi : des clauses concernent le respect par les collaborateurs des lois et règlements (boycott, concurrence, etc.), les conflits d'intérêts, la corruption, etc ${ }^{1164}$.

Ces droits mettent directement en œuvre les droits sociaux fondamentaux identifiés au niveau international. L'entreprise garantit les droits fondamentaux bien qu'elle ne soit pas liée par les textes internationaux. Cette

1158 http://www.icftu.org/displaydocument.asp?Index=991216332\&Language=EN et Renée-Claude DROUIN, International framework agreements: a study in transnational framework agreements: a study in transnational labour regulation, thèse, Montréal, p. 1.

1159 Par exemple le code de General Electric dans les années 80 suivie ou celui de Levi-Strauss en 1991 créé sous la pression publique.

1160 Bernard TEYSSIE, "La négociation collective transnationale d'entreprise ou de groupe ", Droit Social, $1^{\mathrm{er}}$ novembre 2005, $\mathrm{n}^{\circ} 11$, pp. 982 -990.

1161 Rapport à la Commission européenne, La responsabilité sociale des entreprises et la sécurité et la santé au travail, Luxembourg, OPOCE, 2006.

1162 OCDE, http://www.oecd.org/document/12/0,3343,en_2649_34135_35532108_1_1_1_1,00.html (consulté le 26 novembre 2009).

1163 Peter WILKE, Kim SCHÜTZE, Background Paper on International Framework Agreements for a meeting of the Restructuring Forum devoted to transnational agreements at company level, Hamburg, 2 juin 2008, p. 8. V. également Julien BURDA, supra.

1164 Paul-Henri ANTONMATTEI, Philippe VIVIEN, Chartes d'éthique, alerte professionnelle et droit du travail français : état des lieux et perspectives? Rapport au gouvernement, Collection Des rapports officiels, janvier 2007, La documentation française. 
protection concurrente de celle de l'État a pu amener à se poser la question de l'éventuelle déconstruction du droit social. En d'autres termes, le recours à des droits fondamentaux peu protégés et non mis en œuvre juridiquement pourrait conduire à écarter le droit du travail des États, dit droit dur, dans lesquels l'entreprise est installée et desquels elle risquerait de partir. Mais cette question revient à celle de l'impact du droit dur sur le droit mou, ou en d'autres termes, à celle de l'effectivité du droit mou. C'est justement ce qui est remarquable: la RSE connaît une mise en œuvre relativement efficace.

\section{B. Une mise en œuvre de droit mou relativement efficace}

Le fait que les entreprises pratiquant une politique de RSE cherchent à éviter au maximum les tribunaux ne les empêche de prévoir à la fois des mécanismes de mise en œuvre des codes et de règlements des différends en cas de litige.

\section{1) Des procédures de mise en œuvre}

Plusieurs codes de conduite instaurent une mise en œuvre des dispositions protégées ${ }^{1165}$. Par exemple, certains codes prévoient une procédure de monitoring : la mise en œuvre ne se limite pas à l'affichage de panneaux sur les lieux de travail, mais donne un rôle avéré aux syndicats nationaux et locaux. On assiste à un processus de dissémination des règles par le biais des partenaires sociaux. Ils prennent un rendez-vous annuel afin de s'assurer de la bonne mise en œuvre de l'accord. Une logique procédurale fondée sur des modes de représentation dans des comités paritaires locaux relève de cette méthode ${ }^{1166}$. Les dispositions peuvent être très précises et très innovantes.

Il est alors intéressant de se pencher sur l'articulation entre les dispositions du code et le droit des États dans lesquels l'accord trouve à s'appliquer. Le plus souvent, une mise en œuvre ne sera pas nécessaire parce que le code sera valide du fait de l'engagement unilatéral de l'employeur. Parfois, au contraire, il donne lieu à des obligations contractuelles ${ }^{1167}$. Enfin, il peut arriver que les comités paritaires se voient confier la charge de la corrélation.

Cependant, parce que le pouvoir de contrôle des comités n'est pas certain, l'accord risque d'être privé de toute réalité. Afin d'éviter cette inefficacité, il doit être directement applicable dans le pays ou transposé par les syndicats du

\footnotetext{
1165 Jean-François RENUCCI, «Dialogue social et négociation collective à l'échelle communautaire. Constat : les temps européens ne sont pas les temps des entreprises ", Droit social, $2008, \mathrm{n}^{\circ} 1$, pp. $52-56$.

1166 Exemple de Arcelor, accord santé, Marie-Ange MOREAU, «Négociation collective transnationale : réflexions à partir des accords-cadres internationaux du groupe Arcelor Mittal », Droit social, 2009, n 1, pp. 93-102.

1167 V. notamment Charles NEAU-LEDUC, " La responsabilité sociale de l'entreprise : quels enjeux juridiques? », Droit social, n 11, 2006, pp. 952-958.
} 
pays ${ }^{1168}$. Ensuite, une procédure doit permettre de s'assurer de sa mise en œuvre. Plusieurs techniques, plus ou moins précises, ont vu le jour. L'accord peut premièrement contenir des dispositions vagues en matière de procédure. Se pose alors la question de savoir s'il aura un effet obligatoire ou volontaire. Par exemple, l'accord de Rhodia et la Charte sociale de Suez ne contiennent aucune disposition concernant les procédures de mise en œuvre. Il peut ensuite être prévu que le management local sera seul en charge de la mise en œuvre ${ }^{1169}$. La mise en œuvre peut, en troisième lieu, être conjointe, alors entre les mains du management local et des représentants des salariés sans qu'aucune autre précision ne soit donnée ${ }^{1170}$. D'autres accords sont encore mis en œuvre par le biais d'une négociation collective ${ }^{1171}$. Enfin, de nombreux accords précisent qu'ils doivent être mis en œuvre dans les différentes branches de l'entreprise ${ }^{1172}$. Selon Renée-Claude Drouin, une autre classification partage les différents mécanismes entre procédures de mise en œuvre inspirées de techniques managériales, mécanismes paritaires de suivi et procédures de plainte en cas de violation des engagements de l'entreprise. Toujours est-il qu'il en ressort une impression de décentralisation de la décision. Celle-ci est à double tranchant: elle permet une plus grande adaptation de l'accord à la situation locale, mais cette flexibilité peut aussi être son point faible. C'est donc une image nuancée qui ressort de ce panorama. La mise en œuvre des obligations procédurales reste très variable ${ }^{1173}$.

\section{2) Des mécanismes de règlement des différends}

Les systèmes de règlement des différends présentent aussi un intérêt considérable : ils permettent d'éviter le juge extérieur tout en tentant de trouver une solution au litige et en alertant la hiérarchie de l'entreprise d'un nonrespect. Bien que plusieurs codes n'en contiennent pas, il semble que la tendance soit aujourd'hui à une multiplication de ce genre de clauses. De plus en plus, les codes contiennent des techniques de médiation, de résolution du litige qui sont des techniques non judiciaires. Il est intéressant de remarquer que le juge est totalement absent de ce catalogue.

Une voie plus novatrice et directement rattachée aux accords-cadres internationaux tient à l'internalisation des conflits. L'organisation du règlement des conflits en interne peut être plus ou moins construite. Elle est parfois utilisée dans les entreprises n'ayant leur activité que sur un territoire, mais elle présente un intérêt particulier dans les situations transnationales. Ont été

\footnotetext{
1168 C'est le cas de l'accord de Total Platform de 2004. V. Mélanie SCHMIDT, Restructuring and anticipation dimension of existing transnational agreements, Analysis and overview table, rapport pour la Commission européenne, Mai 2008.

1169 V. les accords Arcelor, EADS 2005, Generali.

1170 V. l'accord Renault.

${ }^{1171} \mathrm{~V}$. les accords PSA et EDF.

1172 V. les accords Generali, Suez 1998, Danone 1992 et Lukoil.

1173 Laura R. PRICE, "International Framework Agreements : A Collaborative Paradigm for Labor Relations", in Olivier de SCHUTTER, Transnational corporations and human rights, Oxford ; Portland, Or. : Hart Pub., 2006, pp. 241-260.
} 
recensées à ce jour cinq hypothèses d'internalisation des conflits par les entreprises multinationales. Elles ont vocation à prévenir un procès par le règlement d'un différend au sein de l'entreprise. La procédure instituée peut avoir plusieurs volets. Elle consiste, dans tout les cas, au minimum, en l'octroi d'un droit reconnu à tout salarié d'informer l'entreprise de tout comportement violant l'accord ${ }^{1174}$. Lors de l'identification d'un litige, celui-ci sera pris en charge par des membres désignés au sein de l'entreprise. Les représentants du comité d'entreprise européen signataire peuvent être chargés, en collaboration avec le management de régler les plaintes ${ }^{1175}$. Il peut encore s'agir d'un comité global ou "joint global committee ». Cette hypothèse est généralement préférée lorsque des syndicats internationaux ou des fédérations européennes sont signataires. Elles sont alors chargées du règlement des différends en collaboration avec le management. Un troisième volet procédural consiste en la mise en place d'un panel d'experts indépendants. Ces organismes ad hoc regroupent généralement, encore une fois, les syndicats et le management ${ }^{1176}$. Il peut aussi être fait appel à des organisations non gouvernementales. On assiste alors au passage d'un dialogue social bilatéral à un dialogue civil trilatéral ou trialogue ${ }^{1177}$. Enfin, la dernière solution tient à l'établissement d'un comité paritaire. On pourrait imaginer dans le meilleur des cas une participation d'une organisation internationale. Par exemple, l'Organisation internationale du travail pourrait nommer un responsable chargé de trancher les conflits. Sa dimension institutionnelle lui donnerait une véritable légitimité. Cette dernière hypothèse n'a cependant jamais vu le jour. Dans tous les cas, l'intérêt de ces procédures relève d'une implication directe des partenaires sociaux. Elle permet l'appropriation collective des procédures et des règles de l'accord-cadre. Le management de l'entreprise s'assure ainsi de l'évitement d'une justice nationale ou arbitrale. Dans le même temps, les plaintes des salariés sont reconnues et donnent lieu à un traitement procédural interne. Il faut néanmoins relativiser l'apport de ces mécanismes. Si la présence des syndicats est de nature à garantir une protection des travailleurs, on peut cependant craindre que ces acteurs aient un rôle moins affirmé que le management et un poids inégal. Les sorties de conflits peuvent cependant être le fruit de négociations et de compromis, négociés à un niveau plus central que le litige originel souvent local ${ }^{1178}$. Ce déplacement géographique peut être particulièrement important lorsqu'il s'agit de garantir des droits fondamentaux. Les procédures internes ne permettent cependant pas toujours d'engager l'entreprise sur le terrain d'une entière « responsabilité sociale». La saisine du juge entraîne nécessairement le risque d'une interprétation des termes de l'accord.

1174 V. les accords Renault.

1175 V. les accords EADS, Generali, Suez en 2007 et Total en 2004.

1176 V. les accords Ford 2000, Danone 2001.

1177 Israel DE JESUS BUTLER, « Non-governmental Organisations Participation in the EU LawMaking Process, the Example of Social Non-Governmental Organisations in the Commission, Parliament and Council », 2008, 14, European Law Journal, 5, p. 558 et s.

1178 V. Renée-Claude DROUIN, International framework agreements : a study in transnational framework agreements: a study in transnational labour regulation, thèse, Montréal, p. 1. 
En cas d'épuisement des procédures au sein de l'entreprise, afin d'éviter encore le juge, il est souvent proposé de recourir à l'arbitrage. Au lieu des clauses attributives de juridictions, on trouve alors des clauses d'arbitrage tendant à soustraire un litige actuel (compromis) ou éventuel (clause compromissoire) aux juridictions étatiques pour les confier à un tribunal arbitral. Les parties dérogent ainsi aux règles de compétence judiciaire internationale. La clause est appréciée de façon autonome par rapport au contrat international dans lequel elle est stipulée et est valable en soi sans qu'il y ait lieu de rechercher au préalable la loi applicable ${ }^{1179}$. La clause d'arbitrage peut aussi renvoyer à des modes de règlement para-juridictionnels ${ }^{1180}$.

Si les entreprises semblent de plus en plus résolues à s'engager sur le terrain de la RSE, il est intéressant de constater que les autorités publiques nationales et internationales les regardent d'un œil suspicieux. Parce que ces pratiques ne trouvent pas nécessairement de mise en œuvre efficace et surtout parce qu'aucun tiers n'est chargé de s'assurer de ses bons résultats, les États restent méfiants. Ils cherchent à prendre part au jeu en proposant des encadrements de la RSE. Ils essaient avec difficulté d'instaurer un système d'opposabilité indirecte des droits sociaux.

\section{Une opposabilite indirecte de droit dur}

L'intérêt des États et des organisations internationales pour l'encadrement des pratiques de responsabilité sociale des entreprises a grandi à mesure que celle-ci trouvait sa place dans les entreprises elles-mêmes. La question se pose de s'assurer de l'effectivité de ces pratiques ${ }^{1181}$. Parce que l'entreprise est juge et partie lors de litiges avec ses salariés, les acteurs nationaux et internationaux ont voulu interposer un arbitre extérieur, un tiers et poser des limites. C'est une protection indirecte qui est proposée aux salariés des entreprises multinationales, mais une protection qui devrait être plus efficace parce que de droit dur. Deux techniques se sont répandues : d'un côté, certains États et juges nationaux ont cherché à appliquer leur droit national existant, voire à le détourner pour prendre en compte la RSE (A). De l'autre, plusieurs organisations internationales ont cherché à établir des modèles d'encadrement de ces pratiques (B).

\footnotetext{
1179 Yvon LOUSSOUAM, Pierre BOUREL, Pascal DE VAREILLERS-SOMMIERES, Droit international privé, 8ème édition, Droit Privé, Précis, Dalloz, Paris, 2004, p. 61 et s., spéc. p. 614.

${ }^{1180}$ On pense par exemple aux aides aux résolutions de litiges de l'OIT. Ils ne seront pas pris en compte dans cette étude.

1181 Isabelle DESBARATS, «L'entreprise à l'épreuve du développement durable : complexité et ambiguïté du concept de RSE », Revue juridique de l'environnement, 01/01/2007, pp. 175 -190.
} 


\section{A. Le rôle de l'État : Des stratégies d'application ou de détournement des normes existantes}

Si certains États comme la France ont créé de nouveaux instruments juridiques pour prendre en compte la RSE tels que la loi sur les nouvelles régulations ${ }^{1182}$ ou un devoir posé aux travailleurs d'alerter la hiérarchie en cas d'atteinte aux libertés publiques des salariés ${ }^{1183}$, la plupart d'entre eux ont simplement cherché à appliquer le cadre de droit dur existant. C'est ainsi le juge qui s'est vu donné un rôle de création et de renouvellement des normes afin de les appliquer à des situations auparavant inconcevables. Il intervient lorsqu'un litige est porté à son attention dans le pays d'origine de l'entreprise ou encore dans celui où elle est installée. Si le juge se reconnaît compétent, la question est alors posée de la responsabilité de l'entreprise. Elle peut être pénale, civile ou contractuelle. Si les exemples ne sont pas nombreux, ils présentent l'intérêt de montrer les différentes techniques d'appréhension par le droit dur des pratiques de droit mou.

\section{1) Responsabilité pénale}

Les affaires Total et Unocal ont eu un écho important en Belgique, en France et aux États Unis. En Belgique, la plainte se fonde sur la loi belge du 16 juin 1993 relative à la répression des violations graves au droit international humanitaire telle qu'amendée par la loi du 10 février 1999. Cette loi, dite de compétence universelle, dispose que les juridictions belges sont compétentes pour connaître des infractions prévues " indépendamment du lieu où elles ont été commises ${ }^{1184}$ pour poursuivre les auteurs de crimes contre l'humanité commis même à l'étranger. Une procédure complexe a cependant conduit au dessaisissement des juges.

En France, une plainte à été déposée le 9 octobre 2002 sur le fondement des articles 113-2 et 113-6 du code pénal. Ces articles étendent l'application de la loi pénale française aux infractions commises sur le territoire de la République ", et ce y compris " dès lors qu'un de ses faits constitutifs a eu lieu sur ce territoire». L'article 113-6 dispose que la loi pénale française "est applicable à tout crime commis par un Français hors du territoire de la République. Elle est applicable aux délits commis par des Français hors du territoire de la République si les faits sont punis par la législation du pays où ils ont été commis". Elle a cependant donné lieu à une ordonnance de non-lieu prononcée le 22 juin 2006.

\footnotetext{
1182 Loi 2001-420 du 15 mai 2001.

1183 Paul-Henri ANTONMATTEI, Philippe VIVIEN, Chartes d'éthique, alerte professionnelle et droit du travail français : état des lieux et perspectives? Rapport au gouvernement, 2007, la documentation française.

1184 Olivier de SCHUTTER «Les affaires Total et Unocal, Complicité et extraterritorialité dans l'imposition aux entreprises d'obligations en matière de droits de l'Homme ", Annuaire français de droit international LII, Paris, CNRS Éditions, 2006, p. 61 et s.
} 
Aux États-Unis, le juge s'est appuyé sur l'Alien tort act ${ }^{1185}$. Cette loi permet aussi aux étrangers de venir devant ce fort fédéral avec une action en tort (de droit civil et non pas de droit pénal) pour faire une demande de réparation civile en cas de violation du droit des gens ${ }^{1186}$. Elle était originellement peu appliquée et surtout dans des cas de piraterie. Elle a été ressuscitée en 1980 pour offrir un fort à des personnes étrangères, victimes de torture et plus récemment contre une entreprise privée. Un grand nombre de recours ont été déposés et ce dans deux domaines: la protection de l'environnement et la violation de normes sociales. On trouve par exemple un contentieux mené par des travailleurs sur une plantation au Libéria ${ }^{1187}$. Ils faisaient valoir qu'ils étaient victimes d'esclavage du fait de leurs mauvaises conditions de travail. Si leur plainte n'a pas abouti, il semble néanmoins que les recours sur ce fondement se multiplient.

La technique proposée dans ces différentes affaires consiste d'abord à sortir du ressort juridictionnel du pays et à trouver un fort étranger prêt à accueillir la requête. Il faut ensuite qu'un droit différent de celui du pays du dommage soit appliqué. Les conditions sont nombreuses et difficiles à remplir. Il faut encore noter que ces recours sont fondés sur des droits de l'Homme et non pas sur des droits sociaux ou encore moins sur des droits du travail. Une approche générale pourrait conduire à considérer que l'interdiction de l'esclavage ou encore le droit à la dignité conduit à protéger les droits sociaux des personnes ${ }^{1188}$, mais cette interprétation n'a pas été confirmée et même ces affaires donnent le plus souvent lieu à des jugements nuancés ou encore à des non-lieux.

\section{2) Responsabilité civile 1189}

Un engagement unilatéral, une règle coutumière ou un accord collectif ont pu constituer les fondements d'obligations posées par un accord-cadre à une entreprise multinationale.

En droit civil comme en droit de la Common law, un engagement unilatéral ou un gentleman agreement peut créer des obligations justiciables. Dans ce cas, une entreprise serait sanctionnée si elle s'engage à respecter certains standards de l'accord pour ensuite ne pas s'y tenir. Cette hypothèse a

\footnotetext{
1185 Ibid, p. 61.

1186 V. Horatia MUIRWATT, «La compétence universelle et son incidence sur les droits sociaux fondamentaux", in Marie-Ange MOREAU, Justice et mondialisation du droit du travail, Conférence organisée en collaboration par l'université Paris I Panthéon-Sorbonne et l'IUE, à paraître 2010.

1187 V. http://www.stopfirestone.org/2005/12/alien-tort-claims-act-socialfunds/.

1188 Claire MARZO, « Controverses doctrinales quant à la protection des droits sociaux par la Cour européenne des droits de l'Homme ", Cahiers de droit européen, juin 2010, n¹/2, à paraître ; v. aussi Diane ROMAN, " les droits civils au renfort des droits sociaux...", supra.

1189 Il peut sembler étrange de distinguer entre responsabilité civile et responsabilité contractuelle puisque la première inclut la seconde, mais le passage par les solutions anglo-saxones invite à distinguer entre deux procédés distincts. La responsabilité délictuelle n'est pas mentionnée ici parce qu'elle ne trouve pas d'application concrète.
} 
trouvé à s'appliquer dans l'affaire Kasky contre Nike ${ }^{1190}$. En l'espèce, une déclaration unilatérale a été considérée comme liant son auteur par une technique de droit commercial tenant à la publicité mensongère. Les parties ont allégué un vice dans le consentement du contrat. Cette notion, utilisée en droit de la consommation ${ }^{1191}$, présente l'inconvénient de transposer le contentieux du droit social au droit de la consommation. En effet, le requérant n'est non plus le travailleur, mais le consommateur.

C'est encore ce fondement qui a conduit la Cour de Cassation espagnole dans un arrêt Banco de Espana du 7 mars 2007 à juger que le code de conduite qui obligeait les travailleurs à faire un bilan sur leurs valeurs personnelles dans un objectif de lutte contre la corruption devait respecter les droits fondamentaux des travailleurs ${ }^{1192}$. Plus précisément, il a été dit que le code de conduite relève du pouvoir de direction de l'employeur, qu'il n'a pas à être négocié avec l'organisation syndicale s'il n'existe pas une obligation légale de négocier, mais que ce code doit respecter les droits fondamentaux des travailleurs. Il a enfin été précisé que le non-respect d'un code de conduite, bien qu'il soit un instrument unilatéral, peut engendrer des sanctions disciplinaires conformément au statut des travailleurs.

\section{3) Responsabilité contractuelle}

Une "contractualisation de l'éthique" trouve aussi sa place dans les mécanismes d'encadrement de la RSE. La contractualisation qui passe par les contrats commerciaux conclus par l'entreprise et ses cocontractants ou encore la simple qualification de contrat par le juge saisi d'une affaire de non-respect d'un code de conduite.

C'est cette construction juridique qui a été au fondement de l'affaire Wal Mart aux États-Unis et en Allemagne ${ }^{1193}$. Cette valeur impérative a permis à des travailleurs chinois, bengali, indonésiens et nicaraguayens d'attaquer, le 13 septembre 2005, l'entreprise multinationale Wal-Mart devant la Cour de Los Angeles aux États-Unis en alléguant que cette entreprise n'avait pas respecté les obligations posées par son code de conduite envers les salariés des entreprises sous-traitantes. La question se posait de savoir si ce code pouvait être considéré comme un contrat ${ }^{1194}$. Wal-Mart avait en effet obligé ses sous-

\footnotetext{
1190 Etats Unis, US. Supreme Court, 3 april 2002, Marc Kasky v. Nike, Inc., 02 C.D.O.S. 3790, N S087859.

1191 V. André SOBCZAK, "Legal dimensions of International framework agreements in the field of corporate social responsibility", Relations Industrielles/Industrial Relations, 2007, 62(3), 466-491, p. 477.

1192 Espagne, Corte Suprema de Justicia, 7 mars 2007, Banco de Espana, caso Sentencia n 27595.

1193 Etats Unis, US. District Court for the Central District of California, 30 mars 2007, Jane Doe I, et al., v. Wal-Mart Stores, Inc., Case NO. CV 05-7307 AG (MANx) ; Commentaire : Cynthia ESTLUND, "Something Old, Something New: Governing The Workplace By Contract Again ", (Winter 2007) 28 Comparative Labor Law \& Policy Journal, p. 351 et s.

1194 Ken K. KENNY, "Code or Contract: Whether Wal-Mart's Code of Conduct Creates a Contractual Obligation Between Wal-Mart and the Employees of its Foreign Suppliers", Northwestern Journal of International Law and Business, 2007, n² 27, p. 453.
} 
traitants à signer ce code de conduite, mais aussi à l'afficher dans la langue du pays dans tous les centres de production. Les requérants soutenaient que WalMart avait en retour une obligation de s'assurer que les conditions de travail satisfaisaient à ce code. Malgré l'intérêt qu'avait suscité ce recours, la solution apportée par le juge a été limitée puisqu'elle s'est traduite par un non-lieu. Il faut donc en conclure que le code n'a pas la même valeur qu'un contrat.

Au-delà des tentatives nationales limitées à un territoire défini, plusieurs organisations internationales ont tenté de rendre la protection des droits sociaux dans le cadre de la RSE plus efficace.

\section{B. Le rôle des organisations internationales}

Le rôle des organisations internationales est très limité. L'aveu d'impuissance du bureau international du travail (BIT) est, à ce titre, " glacial » ${ }^{1195}$. Plusieurs raisons se superposent : les difficultés de mise en œuvre ("enforcement") du droit international, le passage nécessaire par les États, l’impossible reconnaissance de la personnalité juridique de l'entreprise en droit international... Elles conduisent à un manque de réactivité de la communauté internationale face aux dangers et aux abus identifiés en matière de RSE.

Les organisations internationales, en attendant une proposition mondiale ${ }^{1196}$, ont pourtant parfois trouvé à s'organiser. On a pu identifier deux tendances : elles peuvent d'abord proposer un texte encadrant les pratiques de la RSE ou servant de modèles aux entreprises souhaitant s'inscrire dans ces procédés. Elles peuvent ensuite participer à la "juridicisation» de la responsabilité sociale.

1) Vers la construction d'un cadre: Des textes d'encadrement des pratiques de la RSE

Parce que le droit international public est applicable ${ }^{1197}$, il faut d'abord penser aux textes classiques de protection des droits sociaux. Par exemple, les conventions de l'OIT ont pu donner lieu à des jugements en faveur de la protection des droits de l'Homme ${ }^{1198}$. Mais, sa portée restreinte a conduit plusieurs organisations internationales à se tourner vers d'autres types de textes.

Le principal problème posé à tous les garants du droit dur national ou international est celui de l'intérêt et de l'adéquation d'un texte encadrant la

\footnotetext{
1195 Disponible sur http://www.iso.org/iso/fr/pressrelease.htm?refid=Ref1299. V. aussi Clotilde DE GASTINES, "ISO 26000 : quand la RSE se veut norme ", Métis, 3 Mai 2010, http://metiseurope.eu/iso-26-000-quand-la-rse-se-veut-norme_fr_70_art_28780.html.

${ }_{1196}$ Manifeste disponible sur http://www.globaleconomicethic.org/02-manifesto-01.php.

1197 Olivier de SCHUTTER, L’incrimination universelle de la violation des droits sociaux fondamentaux, Cellule de recherhe interdisiplinaire en droits de l'Homme, CRIDHO Working Paper 2005/05.

${ }^{1198}$ CEDH, 26 juillet 2005, Silladin c. France, Requête n 73316/01 (esclavage moderne).
} 
RSE. Plusieurs options théoriques sont disponibles : d'abord, la loi peut proposer un cadre obligatoire ou un modèle. Elle peut ensuite poser des obligations matérielles ou procédurales telle que la constitution d'un organe ${ }^{1199}$ ou encore une obligation d'information quant aux pratiques de RSE de l'entreprise. La standardisation est encore une option. Enfin, la coordination complète d'un secteur a aussi été envisagée.

Ces différentes possibilités permettent à un éventail de mesures de voir le jour. De nombreux modèles ont connu un certain essor. En premier lieu, la déclaration de 1998 de l'OIT et les principes directeurs de l'OCDE ont acquis une renommée mondiale. La Déclaration de 1998 de l'OIT a été souvent reprise ${ }^{1200}$. Sa nouvelle approche relative au travail décent semble porter ses fruits $^{1201}$.

De nombreuses procédures de standardisation remportent aussi un grand succès. Ont été imaginées des normes sur les responsabilités des entreprises transnationales et autres entreprises quant aux droits de l'Homme ${ }^{1202}$. Le développement des standards dans ce domaine, comme le Global Compact des Nations Unies, la Global Reporting Initiative, SA 8000, ISO 14001 et bientôt sans doute ISO 26000, poussent de plus en plus à une certaine convergence des pratiques, renforcée encore par l'action des cabinets de conseil et d'audit, des agences de notation extra-financière et de certaines écoles de management ${ }^{1203}$.

L'institution d'obligations trouve un accueil plus mitigé. Par exemple, au sein de l'Union européenne, l'idée a été émise que la RSE n'est pas incompatible avec un cadre juridique. Plusieurs propositions ont stimulé l'intérêt des entreprises ${ }^{1204}$. Mais, aucune législation n'a vu le jour. Le principal problème tient à ce que les entreprises et les autorités communautaires ne réussissent pas à définir la RSE et à lui donner un contenu précis ${ }^{1205}$. Certains souhaitaient utiliser une directive sur le RSE comme un standard minimal fixant ce que les entreprises européennes devraient s'engager à offrir en matière de droits sociaux et environnementaux. D'autres envisagent une loi sur le reporting social et environnemental. L'exemple de la loi NRE française qui oblige les entreprises cotées en bourse à inclure des informations sociales et environnementales dans leur rapport annuel de gestion montre qu'un cadre juridique peut contribuer à

1199 On pensera à l'exemple du comité d'entreprise européen qui, sous l'apparence d'une règle obligatoire, ne propose qu'un mode d'emploi de création du comité qui ne doit pas nécessairement être suivi. V. Directive 2009/38/CE.

1200 V. supra, I.

$1201 \quad$ V. http://www.ilo.org/global/About_the_ILO/Mainpillars/WhatisDecentWork/lang--

fr/index.htm.

1202 ONU, CoDESC, "On the responsibilities of transnational corporations and other business enterprises with regard to human rights ", 13 aout 2003.

1203 André SOBCZAK, "Pour une définition de la RSE à la hauteur des enjeux !", Métis, 16 Avril 2010, http://www.metiseurope.eu/pour-une-definition-de-la-rse-a-la-hauteur-desenjeux_fr_70_art_28765.html

${ }^{1204} \operatorname{COM}(2002) 347$ final.

1205 Régine BARTH, Franzisca WOLFF, Corporate social responsibility in Europe: rhetoric and realities, Cheltenham, Edward Elgar, 2009. 
stimuler les initiatives dans ce domaine, sans pour autant interdire l'innovation ${ }^{1206}$. Il semble pourtant aujourd'hui que ce projet n'ait pas été généralisé.

Une dernière proposition est celle de la coordination complète du droit d'un secteur. Il présente l'intérêt d'annihiler la possibilité d'une entreprise multinationale de se déplacer pour jouer des différents droits nationaux, il rend le mouvement d'une entreprise plus facile et il permet une meilleure application des règles et un meilleur contrôle de celle-ci. Un exemple a pu être apporté par le secteur maritime ${ }^{1207}$. Mais, il pourrait difficilement être reproduit dans d'autres secteurs du fait de l'absence d'un besoin latent de coordination.

Face aux limites de l'encadrement textuel, l'encadrement juridictionnel semble une alternative intéressante.

2) Vers une participation juridictionnelle ou quasi-juridictionnelle des organisations internationales

Au-delà d'une participation réglementaire, les organisations internationales ont tenté d'intervenir d'une façon juridictionnelle ou quasijuridictionnelle. On trouve ainsi une gradation de pratiques des moins aux plus juridictionnelles.

Les procédures les moins juridictionnelles sont entre les mains de l'OIT et de l'OCDE. Les principes directeurs de l'OCDE mettent en place leurs propres systèmes de résolution des litiges tels que des points de contact nationaux ${ }^{1208}$. Le point est actionné là où le dommage nait ou là où il risque de naître ou sur territoire du siège social de l'entreprise mère. Cette procédure évite un blocage par le passage par le pays du litige.

En ce qui concerne la mise en œuvre de la déclaration de 1998, le BIT se limite aujourd'hui à commander des rapports, mais qui n'emportent pas de conséquences. Les techniques sanctionnatrices douces et le suivi propre de la déclaration de l'OIT consistant en une assistance technique ont un impact limité en termes de droit dur, mais c'est peut-être aussi sont intérêt ${ }^{1209}$. L'idée d'une participation accrue de l'OIT comme dernier niveau de recours en cas de différend entre une entreprise et ses employeurs a aussi été mentionnée ${ }^{1210}$. Elle présenterait un intérêt considérable dans la mesure où le BIT est une organisation fondée sur le triptyque États/ salariés/ employeurs. Sa

\footnotetext{
1206 Ibid.

1207 Alexandre CHARBONNEAU, "L'originalité du secteur maritime : interventions judiciaires, règlements alternatifs de conflits et action syndicale internationale " in Marie Ange MOREAU, Justice et mondialisation $d u$ droit du travail, Conférence organisée en collaboration par l'université Paris I Panthéon-Sorbonne et l'IUE, à paraître 2010.

1208 V. http://www.oecd.org/document/3/0,3343,fr_2649_34889_1933123_1_1_1_1,00.html.

1209 V. supra, Julien BURDA.

1210 Marie-Ange MOREAU, « Négociation collective transnationale : réflexions à partir des accordscadres internationaux du groupe Arcelor Mittal », Droit social, 2009, n¹, pp. 93-102.
} 
représentation par tiers lui permettrait d'être un organe de recours privilégié. Mais, la pratique est encore loin de cette avancée.

Plus juridictionnelles sont les pratiques de l'OMC ${ }^{1211}$ ou des Nations Unies. La résolution du Conseil de sécurité 1499 (2003) sur la situation du Congo ou les sanctions économiques des Nations Unies contre des entreprises dans des cas sud-américains et de Rhodésie du Sud sont des exemples intéressants en matière de droits de l'Homme ${ }^{1212}$. La question se pose néanmoins de savoir s'ils pourraient être transposés aux droits sociaux fondamentaux.

Au niveau européen, l'idée d'une compétence universelle en matière civile a été suggérée. Un avant-projet de Convention à La Haye n'a pas abouti1213. Une proposition d'octobre 2008 évoque l'augmentation du champ d'application de la convention de Bruxelles I ${ }^{1214}$ au-delà des litiges européens. L’idée est que les règles de compétence internationale de chaque État doivent disparaître et que des dérogations doivent permettre d'attraire des situations particulières, par exemple si les exigences du procès équitable le requièrent. Cette approche permettrait de sanctionner les violations en matière de droits fondamentaux et une meilleure protection en substance des exigences des droits de l'Homme.

Il faut cependant s'interroger sur l'intérêt d'une juridicisation des pratiques de droit mou. Beaucoup ont remarqué que ce durcissement irait à l'encontre de la nature même de la RSE fondée sur son caractère volontaire ${ }^{1215}$. C'est sur ce croisement paradoxal que le juriste bute aujourd'hui.

\section{Conclusion}

En conclusion, cette étude a été l'occasion de peindre à gros traits le rôle des entreprises, des États et des organisations internationales dans la protection des droits sociaux en matière de RSE. Protection efficace ? La question reste en suspens. S'il apparaît clairement que la RSE se répand inéluctablement, que les entreprises, même de moindre importance, sont directement influencées par ces mécanismes, s'il est désormais admis par toutes les organisations et de nombreux pays que la RSE est à prendre en compte, voire à encadrer, on ne sait toujours pas quelle portée celle-ci peut avoir.

1211 Fabrizio FRANCIONI, "Four ways of enforcing the international responsibility for human rights violations by multinational corporations ", in Marie-Ange MOREAU, Fabrizio FRANCIONI (dir.), La dimension pluridisciplinaire de la responsabilité sociale de l'entreprise, coll. B. Goldman, Presses universitaires d'Aix-Marseille, 2007, pp. 151-171.

1212 John A. ZERK, Multinationals and corporate social responsibility: limitations and opportunities in international law, Cambridge, New York, Cambridge University Press, 2006.

1213 Etienne PATAUT, «Les règles de compétences juridictionnelles en Europe et le rôle du juge à l'épreuve des violations des droits sociaux ", in Marie-Ange Moreau, Justice et mondialisation du droit du travail, Conférence organisée en collaboration par l'université Paris I Panthéon-Sorbonne et l'IUE, à paraître 2010.

1214 Devenu le règlement européen 44/2001.

1215 Bettina LANGE, Socializing Economic Relationships : New Perspectives and Methods for Analysing Transnational Risk Regulation, Centre for Socio-Legal Studies, Conférence 15-16 April 2010, Oxford. 
Sa principale caractéristique de soft law ou droit mou lui appose un sceau de non-droit qu'il est difficile de dépasser. Les mécanismes de droit dur qu'on a tenté de lui superposer se révèlent souvent de faible efficacité du fait de leur inadéquation originelle.

Se pose alors la question de sortir de cette échelle d'analyse allant du droit dur au droit mou. Peut-on imaginer une nouvelle façon de conceptualiser la règle molle sans se référer à la sanction et au juge ? Par exemple, l'impossibilité d'établir de frontière tangible entre le non droit et le droit, l'observation de la procéduralisation du droit du travail au plan mondial, ou encore le constat que l'action et le conflit précèdent le droit ou la norme dévoilent des changements d'approche. Le fait que juge devienne progressivement secondaire amène à sortir de la logique classique, fondée sur la crainte de la sanction judiciaire. Autre exemple, la multiplication des textes de référence en matière de droits sociaux fondamentaux donne une très grande flexibilité aux garants de la loi. Ce constat conduit encore à remettre en question l'application juste et uniforme du droit. Sans apporter une véritable réponse pour le moment, il faut comprendre la responsabilité sociale de l'entreprise comme un sujet nouveau, pluridisciplinaire qui appelle des analyses en matière de droit national et international, de protection des travailleurs, peut-être d'organisation incomplète ${ }^{1216}$ ou encore de théorie de la régulation dans une perspective de la firme construite sur les fondements de l'analyse économique du droit, à partir de la corporate governance. Un détour par les politiques économiques ${ }^{1217}$ ou les techniques managériales ${ }^{1218}$ ou la sociologie pourrait se révéler utiles. Il s'agirait alors de repenser les systèmes de décision au sein des entreprises ${ }^{1219}$.

\section{Pour citer cet article}

Claire Marzo, «La responsabilité sociale des entreprises. A mi-chemin entre la soft law et le jus cogens : la question de l'effectivité de la protection des droits sociaux par les entreprises multinationales», La Revue des Droits de l'Homme, juin 2012 http://revdh.files.wordpress.com/2012/04/la-responsabilitc3a9-sociale-des-entreprises5.pdf

1216 Göran AHRNE, Nils BRUNSSON, N., "L'organisation en dehors des organisations, ou l'organisation incomplète ", AEGIS le Libellio, pp. 1-20, p. 2.

1217 Markus KITZMUELLER, Economics and corporate social responsibility, thèse, Florence, EUI, 2008.

1218 Jan JONKER, Maria DE WITTE, Management models for corporate social responsibility, Berlin, New York, Springer, 2006.

1219 David VOGEL, The market for virtue: the potential and limits of corporate social responsibility, Washington, Brookings Institution Press, 2005. 